MINI-SYMPOSIUM

\title{
Plaque stabilisation by systemic and local drug administration
}

\section{J C Spratt, E Camenzind}

Heart 2004;90:1392-1394. doi: 10.1136/hrt.2004.034975

$C^{1}$ linical manifestations of coronary atherosclerosis are mainly a consequence of one of the following pathogenesis:

- Stable coronary artery plaque without thrombus formation causing variable degrees of luminal narrowing and stable angina

- Superficial plaque erosion, defined as endothelial erosions that may result in thrombus formation and acute coronary syndromes (ACS) (cause $\sim 30-40 \%$ of ACS, more common in younger patients and women) ${ }^{1}$

- Deep plaque rupture defined as fibrous cap rupture with exposed lipid core resulting in in-situ thrombus formation and varying degrees of coronary ischaemia (cause $\sim 60$ $70 \%$ of ACS). ${ }^{2}$

The lack of a linear relation between stenosis severity and the probability of developing an ACS is well recognised. ${ }^{3}$ Indeed the majority of plaques $(\sim 70 \%)$ leading to acute myocardial infarction are not of angiographic significance, implying that factors other than stenosis severity determine the probability of vessel closure. The term "vulnerable" plaque defines the vascular substrate most subject to superficial erosion or plaque rupture which may in turn lead to ACS. While there are multiple complex risk and "trigger" factors at work, accurate identification of a "vulnerable" plaque and efficacious treatment to "stabilise" it, thereby reducing the risk of thrombotic induced acute events, would be of major clinical use. In this review we examine stabilisation and passivation of the "vulnerable" plaque by systemic and local drug delivery (LDD).

Plaque stabilisation can be defined by any intervention or interaction which, by causing a change in either the structure, content or function of an atherosclerotic plaque and/or the overlying endothelium, will either prevent or reduce the severity of erosion or rupture. Plaque passivation is defined as any intervention that decreases the thrombogenicity of the endoluminal oriented vascular surface.

Established risk factors of plaque vulnerability include:

- increased lipid content (>40\%)

- reduced collagen content in a thinned fibrous cap

- increased inflammatory cell infiltration, commonly macrophages

- increased expression of matrix degrading metalloproteinases (MMP)

- reduced expression of tissue inhibitor of MMP (TIMP)

- increased concentrations of macrophage colony stimulating factor (M-CSF)

- haemodynamic shear stress.

Treatment of these risk factors may reduce the probability of plaque erosion or rupture and subsequent ACS.

\section{IDENTIFICATION OF THE VULNERABLE PLAQUE}

Early detection of a vulnerable plaque may allow either systemic treatment or treatment by LDD. Coronary angiography, however, provides limited information on plaque morphology and plaque rupture risk factors. Features of plaque morphology associated with increased plaque vulnerability, as defined by intravascular ultrasound (IVUS) include: eccentric plaque and plaque with significant degrees of echolucency, representing a lipid-rich core and a thin fibrous cap. Clinical studies with IVUS have identified a higher percentage of vulnerable plaques in ACS and two or more plaque ruptures in up to $79 \%$ of cases. ${ }^{4}$

At present imaging modalities such as magnetic resonance imaging using gadolinium derivatives or ferrous oxide particles to detect plaque volume or macrophage intensity respectively, or computed tomography (CT) using liposomal iohexol to reflect plaque volume or ultrafast/electron beam capability to grade plaque calcium, remain a research tool. ${ }^{5}$ Imaging of vulnerable plaques, using infrared spectroscopy and intracoronary thermography, ${ }^{6}$ have demonstrated efficacy in detecting the increased inflammation and metabolic activation seen in an unstable plaque. A further method, "elastography", works by ultrasound reverberation pattern of vascular tissue and can be combined with ultrasound imaging and thermography. ${ }^{7}$

\section{TREATMENT OF THE "'VULNERABLE" PLAQUE Oral Lipid modifying agents}

Statin treatment is established in treating atherosclerosis, and its role in the treatment of the ACS has come under increasing scrutiny. Angiographic studies of statin treatment on coronary artery plaque have shown modest reductions in plaque dimensions, referred to as plaque regression, with proportionately greater clinical benefits, ${ }^{8}$ suggesting a beneficial plaque stabilisation effect which is likely to be multifactorial with:

- diminution of the plaque lipid-rich core

- reduction in inflammation with decreased macrophage and foam cell formation and promotion of fibrous cap thickening

- improvements in endothelial function

- decreasing platelet reactivity.

Abbreviations: $A C E$, angiotensin converting enzyme; $A C S$, acute coronary syndrome; CRP, C reactive protein; Gp, glycoprotein; HDL, high density lipoprotein; IVUS, intravascular ultrasound; LDD, local drug delivery; LDL, low density lipoprotein; LMWH, low molecular weight heparin; MACE, major adverse cardiac events; M-CSF, macrophage colony stimulating factor; MMP, matrix degrading metalloproteinases; $\mathrm{PCl}$, percutaneous coronary intervention; TMMP, tissue inhibitor of MMP; UFH, unfractionated heparin 
Although improvements in endothelial function (with non-specific lipid lowering) can be almost instantaneous, reduction in the incidence of thrombotic events can take up to three months and reduction in the plaque lipid pool up to six months.

Animal studies of cholesterol reduction demonstrate changes in plaque structure including reduction of macrophage numbers and MMP-1 expression and increases in interstitial collagen content resulting in increased plaque stability. ${ }^{9}$ Clinical studies of statin treatment, utilising IVUS, have consistently demonstrated increases in hyperechogenicity index (suggesting an increase in fibrous tissue), reductions in the plaque lipid pool, but only modest reductions in plaque volume. ${ }^{10}$ These changes in plaque structure have translated into improved outcomes in both the MIRACL and the PACT studies of early cholesterol reduction in ACS, ${ }^{11}$ which are likely to relate to beneficial effects on plaque stabilisation, probably by improved endothelial function and possibly by reduced platelet thrombogenicity.

Other cholesterol reducing agents, such as nicotinic acid and ezetimibe, may improve plaque stabilisation by reducing the lipid pool and improving endothelial function, but whether this will extend to improved outcomes in ACS has yet to be established.

Treatments which raise high density lipoprotein (HDL) cholesterol, such as niacin and fibrates, also improve cardiovascular outcomes in high risk groups with low HDL cholesterol, either in combination with simvastatin, or in isolation. ${ }^{12}$

\section{Antithrombotic agents}

An important part of the process leading to arterial thrombotic occlusion is exposure of the subendothelial extracellular matrix, which occurs with either deep plaque rupture or superficial plaque erosion. Exposure of subendothelial tissue introduces platelet adhesion molecules and tissue factors to the circulation, thereby activating the clotting cascade leading to the term atherothrombotic disease. Antithrombotic agents act mainly by reducing intravascular haemostasis, but may also have a direct passivating effect on the vascular wall.

Heparin, both unfractionated heparin (UFH) and low molecular heparin (LMWH), improves outcomes in ACS. The theoretical advantages of LMWH include a prolonged half life and a reduced incidence of thrombocytopenia, and have led in some trials to improved outcomes when compared directly with UFH in patients with ACS. ${ }^{13}$

Aspirin has antiplatelet and anti-inflammatory properties, by covalent binding of cyclo-oxygenase and reducing interleukin-6, C reactive protein (CRP), and macrophage colony stimulating factor. Aspirin has demonstrated benefits both as a stand alone treatment in $\mathrm{ACS}^{14}$ as well as in combination with heparin and clopidogrel. ${ }^{15}$

Adenosine diphosphate receptor antagonists, such as clopidogrel and ticlopidine, inhibit platelet activation, degranulation, and release of prothrombotic and inflammatory mediators, while also preventing activation of the glycoprotein (Gp) IIb/IIIa receptor. These effects have translated into clinical benefit when used in combination with aspirin, ${ }^{15}$ and their rapid onset of beneficial effects may suggest an effect on plaque stabilisation as well as on plaque passivation. ${ }^{16}$

Gp IIb/IIIa inhibitors block the platelet Gp IIb-IIIa receptor, preventing fibrinogen binding and thus platelet aggregation. This class of drugs exhibit their greatest benefit in those patients undergoing percutaneous coronary intervention (PCI). ${ }^{17}$ These beneficial effects may extend beyond inhibition of platelet binding. The Mac- 1 receptor is strongly bound by abciximab, reducing the apoptosis of vascular smooth muscle cells induced by M-CSF activated macrophages. ${ }^{18}$
In addition, as platelets promote the accumulation of inflammatory cells, the antiplatelet aggregation activity of Gp IIb/IIIa inhibitors reduces plaque macrophage burden.

\section{Angiotensin converting enzyme ( $A C E$ ) inhibitors}

ACE inhibitors may improve plaque stability by inhibiting the endothelial dysfunction and oxygen-free radical production caused by angiotensin, while also decreasing macrophage activity and inhibiting smooth muscle cell lipoxygenase activity. Animal studies demonstrating reduced atherosclerosis with ACE inhibitors in non-hypertensive models have led to clinical studies showing improvements in endothelial function and coronary flow-surrogate markers of plaque stabilisation. Observational studies have demonstrated added benefit with ACE inhibitors in ACS out of proportion to the modest degree of blood pressure lowering seen, in patients without significant left ventricular systolic dysfunction, but at high cardiovascular risk. ${ }^{19}$ It could be postulated that this is either due to plaque stabilisation, perhaps in part due to up-regulation of type III collagen synthesis, ${ }^{20}$ or an antiatherogenic effect as suggested by the HOPE study subgroup which demonstrated significant reduction in the rate of carotid intimal medial thickening. ${ }^{19}$

\section{Calcium antagonists}

Calcium antagonists may stabilise plaques by interfering with lipid oxidation and reducing foam cell formation, with the significant increase in transmembrane calcium transport seen in ACS implying an anti-atherogenic role. Amlodipine significantly reduced the rate of carotid intimal medial thickening and reduced ACS admissions in a high cardiovascular risk group. ${ }^{21}$

\section{Other oral agents}

Oral antioxidants may contribute to plaque stabilisation by inhibiting oxidation of low density lipoprotein (LDL) cholesterol and stabilising vascular reactivity-effects most predominant in lipid soluble antioxidants. Despite this studies have consistently failed to show a benefit, ${ }^{13}$ yet diets rich in antioxidants, such as the Mediterranean diet, reduce cardiovascular events, an effect possibly mediated by high concentrations of the $\mathrm{n}-3$ class of essential fatty acids. ${ }^{22}$ Oral treatment with corticosteroids also improves clinical outcomes and reduces in-stent restenosis in patients with high CRP concentrations. ${ }^{23}$ Interestingly, oral administration of immunomodulatory agents (rapamycine) showed no beneficial effect on clinical and angiographic end points. ${ }^{24}$

\section{Local delivery}

Despite the success of oral and intravenous treatment in stabilising the vulnerable plaque, systemic side effects remain an important consideration. In particular antithrombotic agents can be associated with significant bleeding problems. LDD allows the possibility of effective, directed treatment achieving higher site specific concentrations, with much lower doses of drugs, thereby virtually eliminating systemic side effects. LDD may therefore result in more rapid plaque stabilisation and passivation and in addition can be combined with PCI, by either adapted angioplasty catheters or by using stents as a delivery platform.

\section{Local catheter based delivery}

Catheter based delivery allows targeted drug treatment before or after PCI by specially designed catheters, which deliver the compound site specifically into the vessel wall by one of the following principles: (pressure mediated) diffusion; convection or direct injection via micro-needles. LDD of heparin in animal models has greater efficacy than systemic administration and comparable antiplatelet effect 
to systemic Gp IIb/IIIa inhibitors administration. The feasibility of delivering heparin, both UFH and LMWH, has been demonstrated in both an elective clinical setting and during primary angioplasty for acute myocardial infarction. ${ }^{25}{ }^{26}$ Equivalent levels of in-stent thrombosis with LDD are seen, with lower bleeding complications. ${ }^{26}$ Yet although heparin inhibits smooth muscle growth and stimulates endothelial regeneration, with the exception of enoxaparin delivered by the Transport catheter, ${ }^{27}$ this has not translated into lasting clinical benefit in markers such as reduction of restenosis.

\section{Stent based delivery}

Heparin coated stents, where heparin is covalently bound to the polymer coating thereby preventing elution, were developed to reduce stent thrombosis, while reducing or eliminating systemic anticoagulation. The feasibility of using heparin coated stents in combination with oral antiplatelet treatment to reduce acute stent thrombosis was successfully tested by the Benestent II study. ${ }^{28}$ Heparin coated stents have demonstrated utility in other settings, such as in small coronary arteries and vein grafts, but failed to show significant advantages over uncoated stents.

The significant degree of inflammation seen in a vulnerable plaque means that LDD of anti-inflammatory agents holds great potential interest. Reduced in-stent restenosis and a low major adverse cardiac event (MACE) rate, when compared with similar studies, have been demonstrated with a dexamethasone eluting stent in a study group with a high incidence of ACS (40\%). ${ }^{29}$ The study subgroup with ACS showed reduced in-stent restenosis, suggesting that LDD of dexamethasone has a greater effect in vulnerable or inflamed plaques, which may relate to plaque stabilisation. Indeed, local immunomodulation and plaque stabilisation may partially explain the significant reduction of in-stent restenosis and low MACE rates seen with sirolimus eluting stents. $^{30}$

\section{CONCLUSION}

Despite strong evidence for the "vulnerable plaque" concept, some qualifications and unanswered questions exist. It is clear that when assessing the vulnerability of coronary plaque there is a continuum of risk. At one end is the highly vulnerable plaque, with a large lipid-rich pool, a thin fibrous cap, and significant inflammation, and at the other the highly fibrous or calcified plaque with little or no lipid pool. It is in the grey area between that assessment of vulnerability and risk of rupture becomes more difficult. Although many of the commonly used medications in cardiovascular medicine have positive effects on long term plaque stabilisation, the time course of these changes is unclear. Acute plaque stabilisation and passivation may require targeted LLD via a catheter or stent platform with combined treatment of anti-thrombotic and anti-inflammatory/immunomodulating drugs. It seems reasonable to assume that a combined pharmacological approach with local and systemic administration will achieve both optimal early periprocedural efficacy and long term prophylactic efficiency for plaque passivation and stabilisation.

\section{Authors' affiliations \\ J Spratt, Royal Infirmary of Edinburgh, Edinburgh, UK \\ E Camenzind, University Hospital of Geneva, Geneva, Switzerland}

Correspondence to: Dr Edoardo Camenzind, University Hospital Geneva, Rue Micheli-du-Crest 24, 1211 Geneva 4, Switzerland; edoardo.camenzind@hcuge.ch

\section{REFERENCES}

1 Virmani R, Kolodgie FD, Burke AP, et al. Lesson from sudden cardiac death: a comprehensive morphological classification scheme for atherosclerotic lesions. Arterioscler Thromb Vasc Biol 2000;20:1262-75.

2 Fuster V, Badimon L, Badimon JJ, et al. The pathogenesis of coronary artery disease and the acute coronary syndromes. N Engl J Med 1992;326:242-50.

3 Ambrose JA, Tannenbaum MA, Alexopoulos D, et al. Angiographic progression of coronary artery disease and the development of myocardial infarction. J Am Coll Cardiol 1988; 12:56-62.

4 Riofoul G, Finet G, Ginon I, et al. Multiple atherosclerotic plaque rupture in acute coronary syndrome: a three-vessel intravascular ultrasound study. Circulation 2002;106:804-8.

5 Badimon JJ, Fuster V. Can we image the "active" thrombus? Arterioscler Thromb Vasc Biol 2002;22:1753-4.

6 Casscells W, Naghavi M, Willerson JT. Vulnerable atherosclerotic plaque. Circulation 2003;107:2072-5

7 de Korte CL, Schaar JA, Mastik F, et al. Intravascular elastography: from bench to bedside. J Interv Cardiol 2003;16:253-9.

8 Vaughan CJ, Gotto AM, Basson CT. The evolving role of statins in the management of atherosclerosis. J Am Coll Cardiol 2000;35:1-10.

9 Aikawa M, Rabkin E, Okada Y, et al. Lipid lowering by diet reduces matrix metalloproteinase activity and increases collagen content of rabbit atheromas: a potential mechanism of lesion stabilization. Circulation 1998;97:2433-44.

10 Schartl M, Bocksch W, Koschyk DH, et al. Use of intravascular ultrasound to compare effects of different strategies of lipid-lowering therapy on plaque volume and composition in patients with coronary artery disease. Circulation 2001;104:387-92.

11 Schartz GG, Olsson AG, Ezekowski MD, et al. Effects of atorvastatin on early recurrent ischaemic events in acute coronary syndromes. The MIRACL study: a randomized controlled trial, JAMA 2001;285:1711-8.

12 Brown BG, Zhao XQ, Chait $A$, et al. Simvastatin and niacin, antioxidant vitamins, or the combination for the prevention of coronary disease. N Engl J Med 2001;345:1583-92.

13 Cohen M, Demers C, Gurfinkel EP, et al. A comparison of low-molecularweight heparin with unfractionated heparin for unstable coronary artery disease. N Engl J Med 1997;337:447-52.

14 Lewis HD, Davis JW, Archibald DG, et al. Protective effects of aspirin against acute myocardial infarction and death in men with unstable angina. Results of a Veterans Administration cooperative study. N Engl J Med 1983;309:396-403

15 Yusef S, Zhao F, Mehta SR, et al. Effects of clopidogrel in addition to aspirin in patients with acute coronary syndromes without ST-elevation. N Eng J Med 2001;345:494-502.

16 Yusuf S, Mehta SR, Zhao F, et al. Early and late effects of clopidogrel in patients with acute coronary syndromes. Circulation 2003;107:966-72.

17 Boersma E, Akkerhuis KM, Theroux P, et al. Platelet glycoprotein Ilb/Illa receptor inhibition in non-ST-elevation acute coronary syndromes: early benefit during medical treatment only, with additional protection during percutaneous coronary intervention. Circulation 1999; 100:2045-8.

18 Monroe SV, Kerensky RA, Rivera E, et al. Pharmacological plaque passivation for the reduction of recurrent cardiac events in acute coronary syndromes. $J$ Am Coll Cardiol 2003;41:23S-30S.

19 Yusef S, Sleight $P$, Pogue J. Effects of an angiotensin-converting-enzyme inhibitor, ramipril, on cardiovascular events in high-risk patients. The heart outcomes prevention evaluation study investigators. N Eng J Med 2000;342: 145-53.

20 Claridge MW, Hobbs SD, Quick CR, et al. ACE inhibitors increase type III collagen synthesis: a potential explanation for reduction in acute vascular events by ACE inhibitors. Eur J Vasc Endovasc Surg 2004;28:67-70.

21 Pitt B, Byington RP, Furberg CD, et al. Effect of amlodipine on the progression of atherosclerosis and the occurrence of clinical events. Circulation 2000;102:1503-10.

22 de Lorgeril M, Salen P, Martin JL, et al. Mediterranean diet, traditional risk factors, and the rate of cardiovascular complications after myocardial infarction: final report of the Lyon diet heart study. Circulation 1999;99:779-85.

23 Versaci F, Gaspardone A, Tomai F, et al. Immunosuppressive therapy for the prevention of restenosis after coronary artery stent implantation (IMPRESS study). J Am Coll Cardiol 2002;40:1935-42.

24 Brara PS, Moussavian M, Grise MA, et al. Pilot trial of oral rapamycin for recalcitrant restenosis. Circulation 2003;107:1722-4

25 Camenzind E, Bakker WH, Reijs A, et al. Site-specific intracoronary heparin delivery in humans after balloon angioplasty. A radioisotopic assessment of regional pharmacokinetics. Circulation 1997;96:154-66.

26 Esente P, Kaplan AV, Ford JK, et al. Local intramural delivery of heparin during primary angioplasty for acute myocardial infarction: results of the local PAMI pilot study. Cath Cardiovasc Interv 1999;47:237-42.

27 Stefan KR, Pawel B, Jack ML, et al. Local delivery of enoxaparin to decrease restenosis after stenting: results of initial multicenter trial: Polish-American local Lovenox NIR assessment study (The POLONIA Study). Circulation $2001 ; 103: 26-31$

28 Serruys PW, van Hout B, Bonnier $\mathrm{H}$, et al. Randomised comparison of implantation of heparin-coated stents with balloon angioplasty in selected patients with coronary artery disease (Benestent II). Lancet 1998;352:673-81.

29 Liu X, Huang Y, Hanet C, et al. Study of antirestenosis with the BiodivYsio dexamethasone-eluting stent (STRIDE): a first-in-human multicenter pilot trial. Catheter Cardiovasc Interv 2003;60:172-8.

30 Moses JW, Leon MB, Popma JJ, et al. Sirolimus-eluting stents versus standard stents in patients with stenosis in a native coronary artery. N Engl J Med 2003;349:1315-23. 